\title{
EchoGéo
}

42 | 2017

Varia

\section{Lieux et réseaux d'enfermement des migrants à la frontière orientale de la Grèce}

\section{Laurence Pillant}

\section{(2) OpenEdition}

1 Journals

\section{Édition électronique}

URL : https://journals.openedition.org/echogeo/15177

DOI : 10.4000/echogeo. 15177

ISSN : 1963-1197

\section{Éditeur}

Pôle de recherche pour l'organisation et la diffusion de l'information géographique (CNRS UMR 8586)

Référence électronique

Laurence Pillant, «Lieux et réseaux d'enfermement des migrants à la frontière orientale de la Grèce », EchoGéo [En ligne], 42 | 2017, mis en ligne le 31 décembre 2017, consulté le 31 juillet 2021. URL: http://journals.openedition.org/echogeo/15177; DOI : https://doi.org/10.4000/echogeo.15177

Ce document a été généré automatiquement le 31 juillet 2021.

EchoGéo est mis à disposition selon les termes de la licence Creative Commons Attribution - Pas d'Utilisation Commerciale - Pas de Modification 4.0 International (CC BY-NC-ND) 


\title{
Lieux et réseaux d'enfermement des migrants à la frontière orientale de la Grèce
}

\author{
Laurence Pillant
}

1 La frontière orientale de la Grèce devient dans les années 2000 un lieu de franchissement sans autorisation des migrants venus d'Afrique (en majorité d'Algérie et de Somalie), du Moyen-Orient (notamment d'Irak, de Palestine puis de Syrie) et d'Asie (en majorité d'Afghanistan et du Pakistan) dans les années 2000. Peu franchie jusque-là $^{1}$ (Papadopoulou-Kourkoula, 2008), elle est attractive pour des personnes souhaitant se rendre en Europe. En 2010, la police interpelle vingt fois plus de migrants qu'en $2003^{2}$. Avec l'intensification des contrôles en Méditerranée (Schmoll et al., 2015), la localisation géographique proche d'Istanbul à la lisière de l'Union européenne (Pérouse, 2002 ; Bathaïe, 2009) et un important secteur informel (Calavita,2005 ; King et DeBono, 2013), la Grèce confirme sa position de pays d'immigration. L'entrée en vigueur des accords de Schengen en Grèce, l'application du règlement Dublin II $^{3}$ ainsi que le vote de la loi $2910 / 2001^{4}$ contribuent à la mise en place d'une politique migratoire principalement basée sur l'enfermement ${ }^{5}$ des migrants ayant franchi la frontière sans autorisation.

2 Jusqu'en 2011, le franchissement frontalier irrégulier est pénalement répréhensible. En vertu de la loi 2901/2001, le procureur peut décider de suspendre la condamnation pénale au profit d'une expulsion de la personne, une pratique mise en place dans les années 1990 en lien avec une migration albanaise. Les étrangers doivent être placés en rétention jusqu'à complétude du renvoi. Contrairement aux Albanais ${ }^{6}$, les migrants interpellés à la frontière orientale sont difficilement expulsables compte tenu du manque d'accord de réadmission dont la Grèce est signataire ${ }^{7}$, et des difficultés de fonctionnement de l'accord de réadmission avec la Turquie ${ }^{8}$. Cela entraîne des décisions d'expulsions suivies de périodes d'enfermement variables, de quelques jours à plusieurs mois ${ }^{9}$, qui se terminent par des remises en liberté avec une notification de sortie du territoire. 
Cette politique se traduit par l'émergence de lieux d'enfermement dans l'ensemble des localités de la frontière orientale. Le terme de lieu est ici entendu comme un « espace de coprésence dans lequel la notion de distance est abolie" (Sélimanovski, 2009, p. 119-120). Il est façonné par les rapports de coprésence qui le définissent (Baby-Collin, 2014, p. 21). Il est sujet à des transformations permanentes. Bien que dans le cas des lieux d'enfermement, les limites matérielles (barbelés, murs et autres) soient visibles et réelles pour les personnes qui y sont enfermées, l'analyse ne saurait s'y limiter (Milhaud, 2018, p. 57). Les observations et entretiens menés montrent que les liens avec l'extérieur les impactent et qu'une dichotomie trop marquée entre l'intérieur et l'extérieur limite l'analyse (Darley et al., 2013).

Les lieux d'enfermement recensés, au nombre de $56^{10}$ entre 2003 et 2014, ont des formes et des fonctionnements différents tant le cadre qui les régit est peu évocateur jusqu'en 2011 (vote de la loi 3907/2011 ${ }^{11}$ ). À la frontière gréco-turque, ils peuvent cependant être divisés en trois types: les lieux improvisés du début des années 2000 - ce sont des hangars, des entrepôts, ou des hôtels qui sont mobilisés pour l'enfermement -; les lieux institutionnalisés qui sont construits spécifiquement pour l'enfermement à partir de 2005 ; les lieux intermédiaires - commissariats de police, containers, locaux utilisés en l'absence ou en support du lieu d'enfermement principal (illustration 1).

Illustration 1 - Géographie de l'enfermement à la frontière orientale de la Grèce 2005-2012

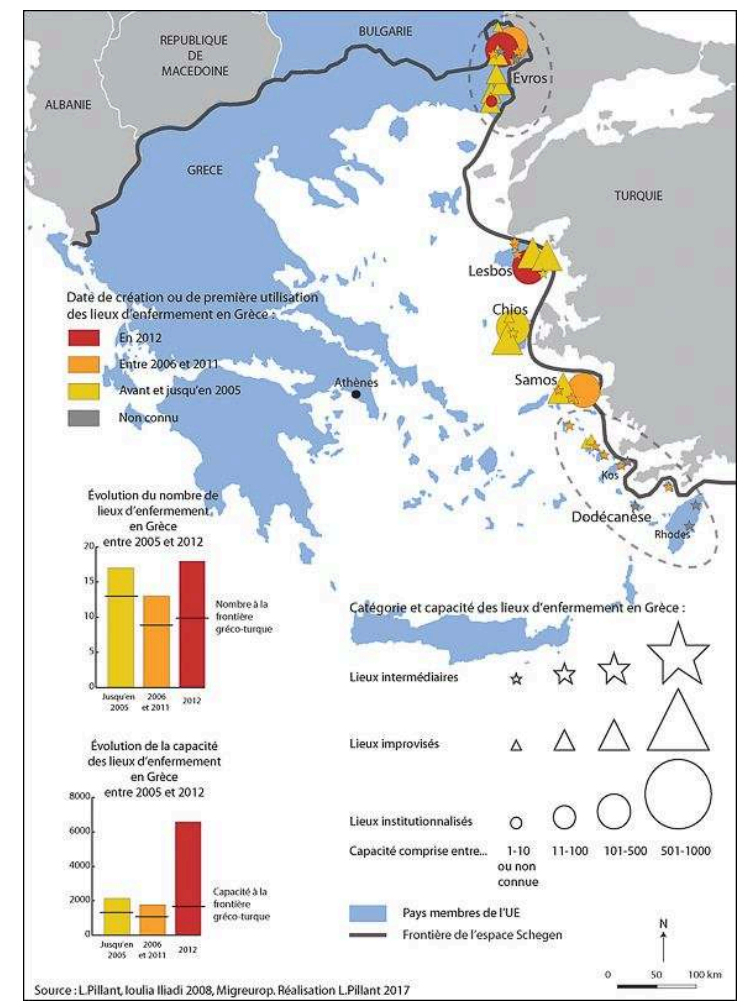

Source : L. Pillant, Ioulia Iliadi, 2008, Migreurop ; réalisation : L. Pillant, 2017.

Tous les récits de migrants recueillis sur leur période d'enfermement dans les années 2000 illustrent une politique migratoire répressive et discrétionnaire dont les modalités varient selon les localités, les lieux d'enfermement, la nationalité des migrants, etc. Dans un travail de doctorat sur le contrôle migratoire à la frontière orientale de la Grèce, j'ai voulu comprendre ce qui façonne l'enfermement, ainsi que la 
manière dont le contrôle des autorités (police et garde-frontière) s'exerce sur les migrants durant cette période.

6 Le travail d'enquête a nécessité une ethnographie de plusieurs mois à la frontière gréco-turque entre 2009 et 2014, des entretiens avec les autorités, avec des intervenants dans les lieux d'enfermement, et les institutions publiques ${ }^{12}$. En l'absence d'observation directe systématique et prolongée dans ces lieux, ces entretiens ont été multipliés, répétés et confrontés aux témoignages des migrants interrogés ${ }^{13}$. Le traitement d'archives du ministère de l'Intérieur ${ }^{14}$, des préfectures de Lesbos, Évros et le Dodécanèse ainsi que les archives de certains lieux d'enfermement ${ }^{15}$ ont aussi permis de compléter le manque d'information disponible sur le sujet.

7 Il s'agit dans cet article de comprendre et d'analyser les modalités de contrôle des autorités sur les migrants au sein des lieux. Pour cela, il est proposé d'explorer la manière dont l'incertitude spatio-temporelle dans laquelle les autorités plongent les migrants lors de la période d'enfermement constitue un puissant facteur de contrôle à l'échelle des lieux et de leur réseau.

\section{L'incertitude spatio-temporelle comme instrument de contrôle dans les lieux d'enfermement}

8 L'analyse de ce qui se déroule dans les lieux d'enfermement montre une multiplicité des pratiques policières. Le cadre législatif qui régit ces lieux est peu précis et si les raisons de la privation de liberté sont sans équivoque, ses conditions sont peu réglementées jusqu'en 2011. Les observations, entretiens et traitements d'archives mettent la lumière sur la confusion spatio-temporelle qui règne dans ces lieux et l'impact déstabilisant sur les migrants. Ainsi, à la contrainte de la suspension du parcours migratoire s'ajoute l'exercice du contrôle des autorités. Si «l'espace-temps [...] n'est pas le conteneur passif de la pratique sociale ou une simple toile de fond contre laquelle se jouent les événements sociaux» (Gill, 2009, p. 186), il s'agit ici de comprendre dans quelle mesure la maîtrise du temps et de l'espace de l'enfermement devient une des modalités de contrôle des autorités sur les migrants, et donc un instrument de pouvoir.

\section{Usage répressif de l'incertitude spatiale au sein des lieux d'enfermement}

L'amélioration des conditions de rétention et la création de lieux institutionnalisés à la frontière gréco-turque à partir de 2005 entraînent l'apparition d'espaces tels que le réfectoire ou encore les espaces dédiés à la promenade, qui n'existaient pas dans les lieux improvisés. Ces nouveaux espaces, pourtant présentés comme des marques de l'amélioration des conditions d'enfermement, peuvent rapidement devenir des espaces de mise à l'écart dans la mise à l'écart.

10 L'exemple du centre de rétention de Vathy ${ }^{16}$ à Samos est à cet égard édifiant. En avril 2010, une trentaine de migrants engage une grève de la faim pour contester le manque d'information sur leur sort ainsi que les conditions d'enfermement. Alors que des manifestations de soutien étaient organisées à l'extérieur du lieu, le terrain de 
basket-ball est utilisé pour confiner exceptionnellement les migrants et leur empêcher toute communication avec les personnes qui manifestent à proximité ${ }^{17}$.

Illustration 2 - Croquis du lieu d'enfermement de Vathy à Samos

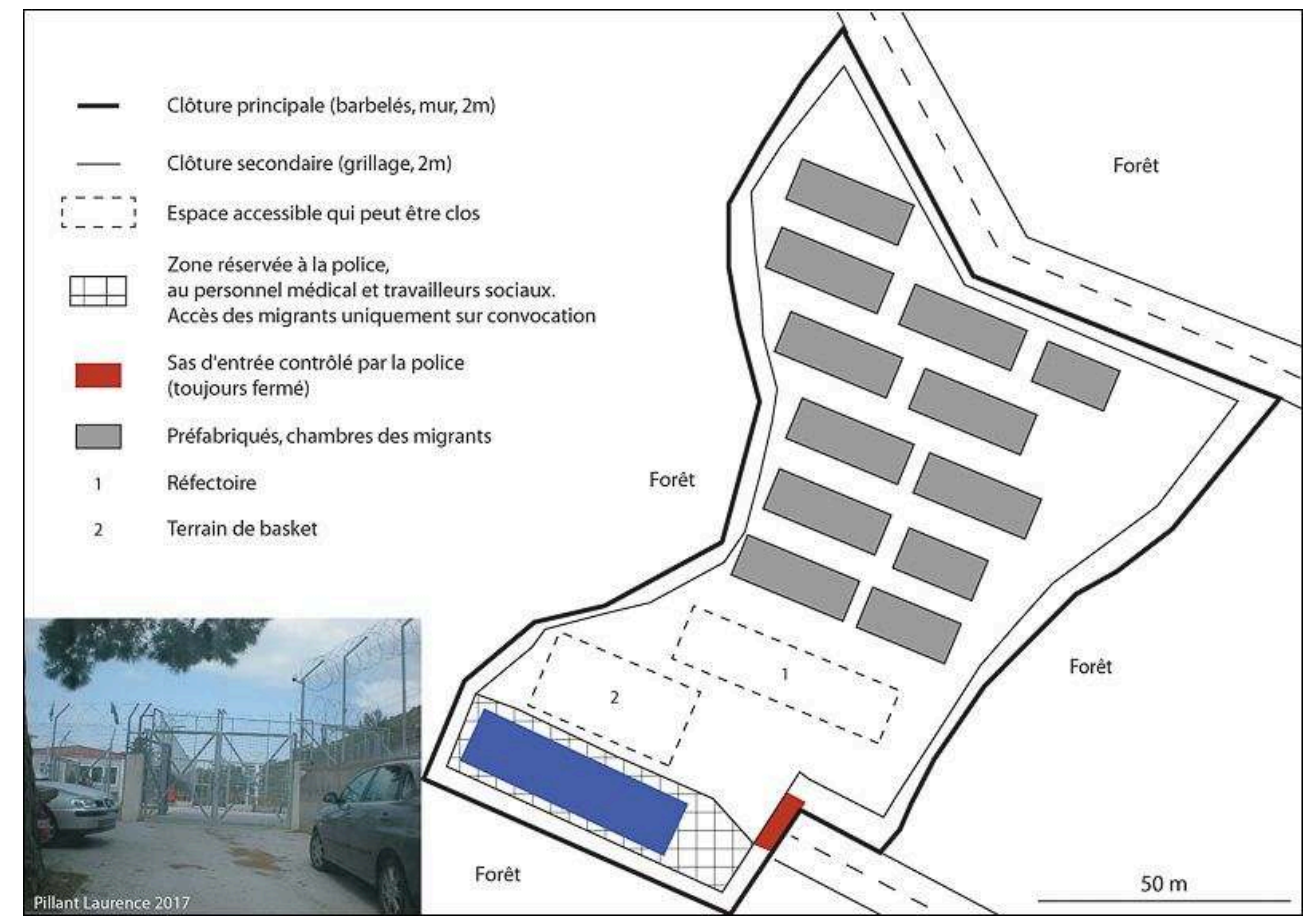

Auteur : L. Pillant, 2017.

11 Ces lieux, présentés dès leur construction comme des espaces plus cléments et d'apparence moins répressive, comme en témoigne la présence d'un terrain de basketball à Vathy, peuvent être utilisés à des fins contraires. Dans les cas avancés de constructions présentées comme respectueuses des migrants, l'accès à une cour extérieure reste soumis à la volonté policière. La surface des aires de libre circulation au sein des lieux peut se réduire de manière radicale en cas de contestation de la part des migrants ou pour les besoins des autorités. Cela entraîne une confusion spatiale au sein des lieux. Ces procédés sont la clef de la mise en œuvre d'une sorte d'enfermement dans l'enfermement dont les autorités disposent arbitrairement pour exercer une pression sur les migrants.

12 Il y a donc bien une maitrise par les autorités de l'espace de l'enfermement dont l'organisation peut être bouleversée: les espaces de liberté peuvent être fermés soudainement, la cour interdite d'accès, le dispensaire peut devenir un espace de mise à l'écart supplémentaire (Pillant, 2014). La confusion dans les repères spatiaux induite par les autorités contribue à donner à ces lieux un caractère répressif, qui se trouve accentué par la maîtrise du temps de l'enfermement.

\section{Usage répressif de l'incertitude temporelle de la durée d'enfermement}

Le temps et sa maîtrise sont l'une des clefs de la compréhension du caractère répressif des lieux d'enfermement. La fréquence de libération et la durée de l'enfermement sont 
des données majeures pour comprendre ces mécanismes. Le contrôle du temps par les autorités accentue la sensation de privation de liberté. Lorsque l'on recoupe les entretiens effectués auprès des migrants à cette frontière, il est frappant de constater la récurrence du manque d'information ou la désinformation dont ils sont l'objet de la part des policiers.

"Je ne savais pas où j'étais, quelle ville. Je savais seulement que c'était la Grèce. Après, quand on est sortis du centre, un ami a trouvé où on était. [...] La police ne nous disait rien. Si, elle nous a dit: "vous allez rester 10 à 15 jours pour qu'on vous fasse des papiers et que vous partiez et que vous soyez libres". On est entré (dans le centre) et on est resté 3 mois " (M. à Mytilène en mai 2014).

14 Ce témoignage est représentatif des différents témoignages recueillis à ce sujet. L'approximation ou la désinformation concernant le temps de l'enfermement représente à la fois une violence faite aux personnes qui se trouvent ainsi dépossédées de leur temps, mais aussi une forme de soumission à l'administration. Les intermédiaires (travailleurs sociaux, psychologues, etc.) viennent ensuite apaiser (Tassin, 2016, p. 41) les tensions inhérentes à ces confusions temporelles. «Le centre est calme, mais la police n'explique rien alors on doit rassurer les gens, leur dire qu'ils ne sont pas là pour la vie » (traducteur de la région d'Évros employé par la police, le 13 juillet 2012).

Repousser la date de libération est aussi un moyen de contrôle qui permet aux autorités de reporter les possibles contestations des migrants après l'annonce du temps de rétention. Mais cette confusion provoque des troubles psychologiques comme le spécifie Médecin sans frontières (MSF) dans un de ses rapports. "Les conditions de détention inacceptables, la peur de l'expulsion, l'ignorance concernant la durée de la détention, l'incertitude quant à l'avenir, les difficultés de communication [...], ont un impact négatif sur la santé mentale des immigrés» (MSF, 2012, p. 6) Les migrants enfermés sont donc dépossédés de leur temps. Ils ne peuvent pas compter sur la régularité des périodes d'enfermement tant ces dernières dépendent de nombreux critères : profil des migrants, effectifs policiers, conjoncture politique, mobilisations locales, intervention extérieure, etc. Il y a par exemple une différenciation du temps de l'enfermement selon la nationalité et par rapport à la vulnérabilité (migrants malades, mineurs, etc.).

«Il y a ceux qui restent d'une semaine à 6 mois, certains autres arrivent le matin et partent le soir. Ça dépend de la nationalité. Les pays qui partent rapidement sont le Pakistan, l'Afghanistan, la Palestine, le Bangladesh. Ils restent quelques jours, trois jours, et ils partent. La Syrie, l'Algérie, ils partent directement. Maintenant, le Maroc, Saint-Domingue, l'Irak, l'Iran, l'Égypte, ils restent entre un mois à 6 mois " (une infirmière, Évros, le 21 juin 2012)

16 Les facteurs qui font varier la durée d'enfermement sont multiples et dépendent entre autres des régimes différenciés selon les nationalités, mais aussi de l'engorgement des services dû à une augmentation du nombre d'interpellations. La tension que génèrent ces différenciations est amplifiée par les erreurs notables d'attribution de nationalité par les autorités, dont les répercussions sont importantes pour les migrants.

17 À la dépossession de soi par l'enfermement (Le Courant, 2010) s'ajoute d'une certaine manière la dépossession de soi par l'erreur d'identification qui éclaire une autre forme d'arbitraire. Même si le manque de formation des agents ou la lenteur de la procédure administrative sont évoqués pour expliquer ces erreurs et incertitudes, les autorités locales ne cachent pas l'usage de l'enfermement comme outil répressif. Ainsi, même dans des localités frontalières aux faibles effectifs policiers et sans lieu d'enfermement, 
retenir les migrants peut être vu comme un enjeu dissuasif, comme en témoigne ici le responsable de la police de Kos rencontré en novembre 2012.

"On a entre 45 heures et 3 jours pour décider si on les laisse libres ou pas, avec le papier qui leur indique qu'ils ont de 7 à 30 jours pour quitter le pays. [...] On relâche tout le monde sauf $10 \%$ en tout (dont $3 \%$ des Syriens), qui vont dans d'autres centres en Grèce pour qu'ils ne croient pas qu'ils peuvent venir comme ça tout le temps. Comme pour faire un exemple » (le responsable du contrôle migratoire au commissariat de Kos, le 16 novembre 2012).

Le caractère dissuasif de la mise à l'écart est récurent dans les discours des autorités. À cet égard, la période d'enfermement instaure un rapport de méfiance des migrants envers les autorités. Au brouillage "des frontières spatiales et sociales " propre à l'enfermement (Darley 2008, p. 395) s'ajoute un brouillage temporel.

Si l'étude des pratiques dans ces lieux montre une confusion du temps et de l'espace comme modalité de contrôle, à l'incertitude au sein du lieu d'enfermement s'ajoute la possibilité de transferts du jour au lendemain.

\section{Géographie du contrôle migratoire : mobilités forcées et enfermement en chaîne}

20 En Grèce, les transferts de migrants, après une arrestation à la frontière, occupent une place importante dans le contrôle migratoire : transfert du port au centre de rétention, d'un commissariat à un autre, etc. Plusieurs motifs de transfert d'un lieu à un autre ont été identifiés: la sanction ${ }^{18}$, l'absence de lieu d'enfermement, leur saturation, ou l'absence des autorités responsables de l'identification dans la zone d'interpellation. Dans leur ensemble, et selon les documents analysés, les transferts de migrants ne font pas l'objet d'une régulation spécifique, et sont décidés exclusivement par les autorités.

21 Ces transferts sont marqués par une continuité de la mise à l'écart et dessinent des réseaux de lieux d'enfermement qui se structurent autour des relations entre acteurs. Pour les migrants, ils sont synonymes d'« itinérance rétentionnaire [...] qui dépossède les migrants de leurs trajectoires " (Michalon, 2012, p.6). Au-delà de l'étude de leur usage disciplinaire par les autorités, qui n'est qu'une des multiples raisons de ces transferts, l'analyse de la tension entre l'enfermement et la mobilité semble fondamentale pour la compréhension des mécanismes de contrôle migratoire (Darley et al., 2013, p. 15). L'étude des réseaux de lieux d'enfermement comporte ici plusieurs objectifs. Elle permet de comprendre la fonction des lieux dans le contrôle migratoire et illustre la manière dont les transferts entraînent une multiplication des espaces de la mise à l'écart. Enfin, elle ouvre une réflexion sur le rôle des acteurs (employés d'ONG, policiers et militants) au sein de ces réseaux et la conséquence de tel réseau pour les migrants.

\section{La fonction des lieux d'enfermement dans les réseaux}

Dans un premier temps, les lieux d'enfermement à la frontière orientale de la Grèce sont connectés entre eux par les transferts de migrants. Les entretiens effectués durant cette recherche n'ont pas pu combler le manque de données officielles concernant ces transferts. L'analyse des archives préfectorales et ministérielles pour des périodes spécifiques ${ }^{19}$ a donc été fondamentale. Ces données couplées à la géographie policière des localités ont permis de schématiser des réseaux de lieux d'enfermement 
(illustrations 3 et 4). Sur les croquis sont représentés les lieux selon la typologie exposée en amont (lieux intermédiaires, lieux institutionnels et lieux improvisés), le commissariat auquel ils sont rattachés, et la localisation de la frontière (représentée schématiquement).

Il existe deux formes de réseaux au regard de telles mobilités: des réseaux multipolarisés avec une multiplication de différents lieux de capacité et de taille similaire, et des réseaux polarisés qui s'organisent autour d'un lieu d'enfermement principal. Les cas de la région d'Évros Sud et Nord sont présentés ici à titre d'exemple. Le sud de la région d'Évros en 2012 offre une organisation particulièrement stable d'un réseau multipolarisée ${ }^{20}$. En effet, depuis les prémisses de la mise en place du contrôle migratoire (dès la première moitié des années 2000), ce sont les mêmes lieux qui sont mobilisés, placés sous la juridiction des mêmes autorités de contrôle. Cette stabilité et la pluralité des lieux existants s'accompagnent d'une spécialisation.

Illustration 3 - Réseau multipolarisé des lieux d'enfermement du Sud de la région d'Évros

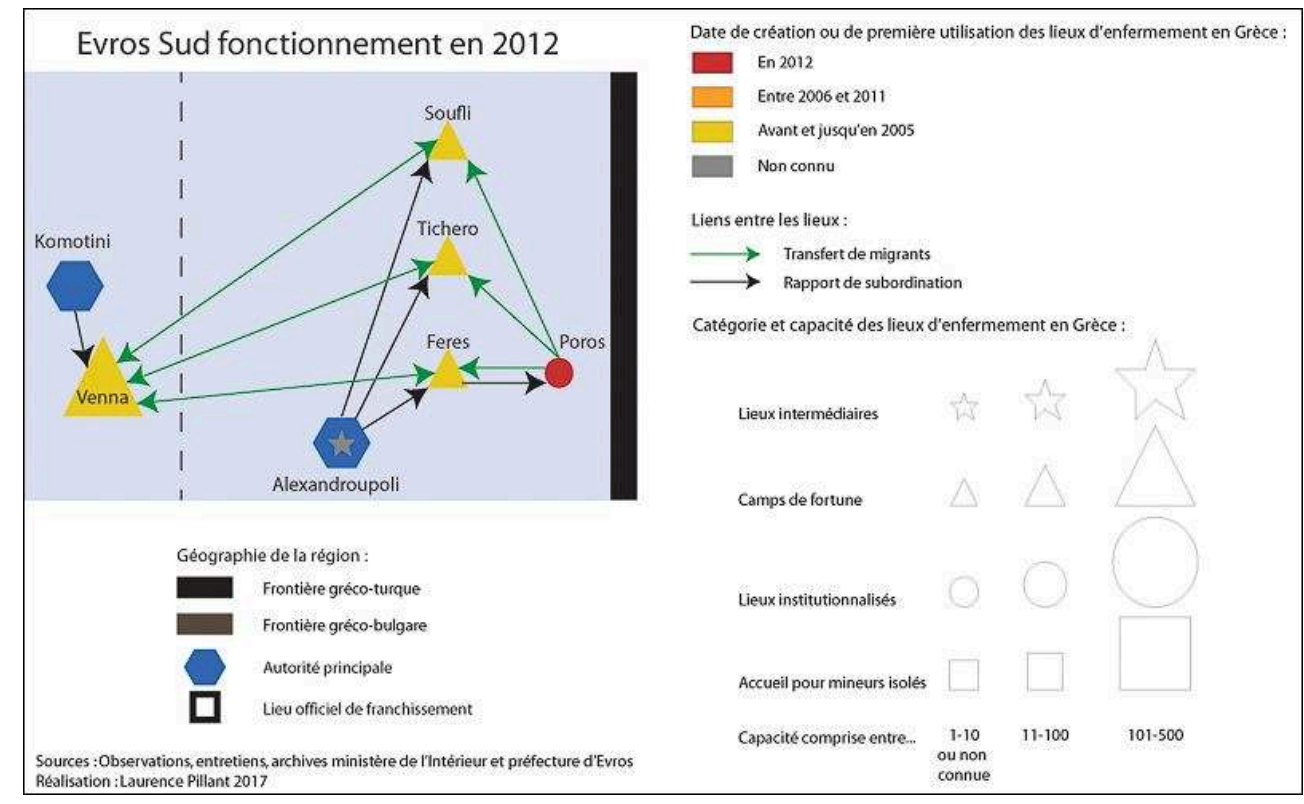

Sources : observations et entretiens, archives du ministère de l'Intérieur et préfecture d'Evros ; réalisation : L. Pillant, 2017.

En 2012, à Poros où sont enregistrées toutes les arrivées, les gardes-frontières et les agents de Frontex identifient les migrants et les redirigent vers les lieux de la région en fonction des places disponibles. Les mineurs étaient dirigés vers Soufli, et les personnes enfermées pour une longue durée à Venna, village particulièrement isolé (à $16 \mathrm{~km}$ de la première ville). Il existe entre ces lieux une répartition des prérogatives très claire, bien qu'officieuse. L'augmentation des arrivées et l'évolution des politiques migratoires n'entraînent pas de bouleversement de ce fonctionnement.

En 2012, la région d'Evros-Nord est organisée selon un réseau polarisé, les mêmes configurations sont observées à Lesbos, Chios et Samos en 2009. 
Illustration 4 - Réseau polarisé des lieux d'enfermement du Nord de la région d'Évros

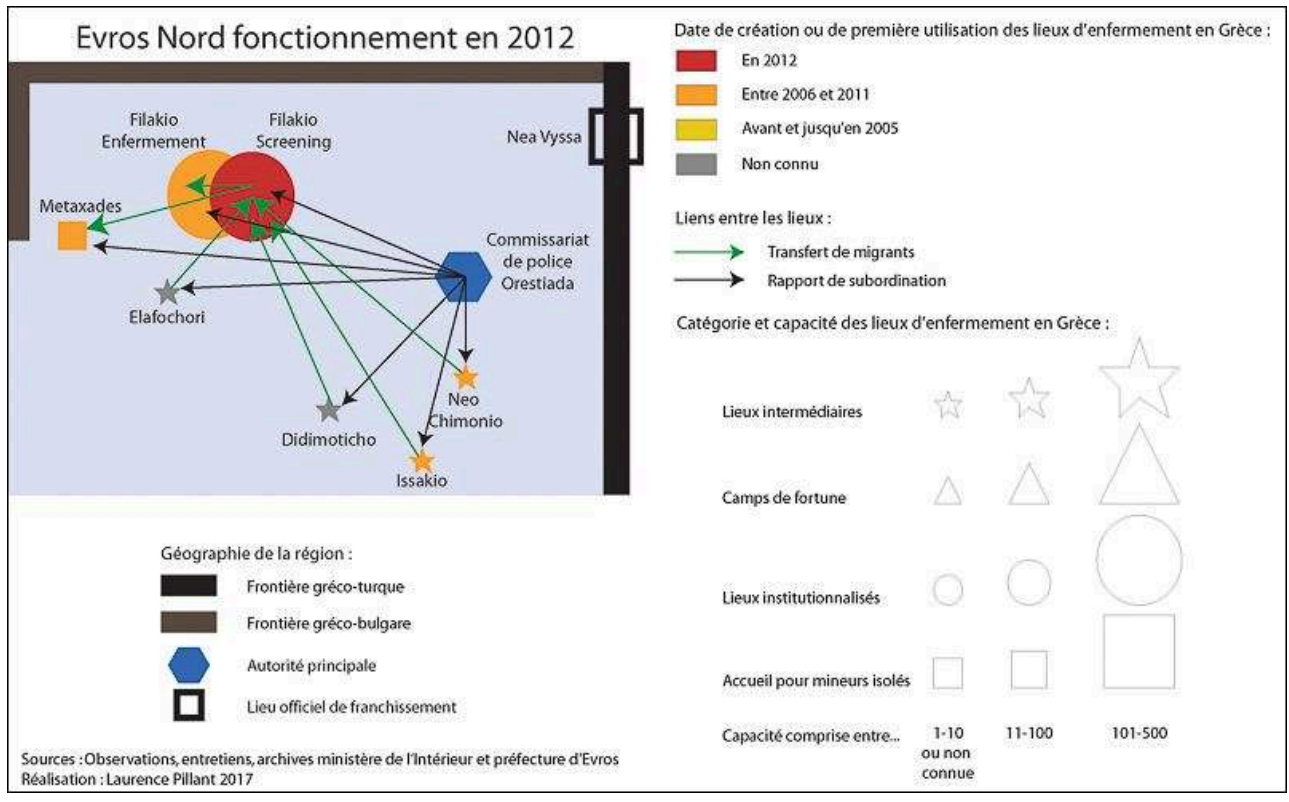

Sources : observations et entretiens, archives du ministère de l'Intérieur et préfecture d'Evros : réalisation : L. Pillant, 2017.

Au sein de ce réseau polarisé, le principal lieu d'enfermement, Filakio, est au centre du fonctionnement du contrôle migratoire et centralise les fonctions d'identification et d'enfermement. En période de nombreuses arrestations et de saturation de Filakio, les gardes-frontières utilisent des lieux intermédiaires, tels que les commissariats d'Isaakio et de Neo Cheimonio. Ces derniers ont alors une fonction d'amortisseurs et se substituent aux lieux d'enfermement institutionnalisés. Au sein de ce type de réseau, la capacité rétentionnaire de la région ne se limite pas au lieu d'enfermement principal et peut être démultipliée jusqu'à ce que les lieux intermédiaires soient complets. Ce fonctionnement est déjà bien ancré historiquement et mobilise des structures qui datent des prémisses de l'enfermement à la frontière. Ce type de réseau pose des problèmes de visibilité des migrants et de légalité des pratiques des autorités. En 2012, un employé de l'UNHCR, responsable de la surveillance des pratiques d'enfermement dans la région, reconnaissait ne pas pouvoir suivre les évolutions de l'utilisation de toutes les structures ${ }^{21}$.

L'enfermement est pratiqué à la frontière gréco-turque avec une temporalité et des modalités différentes selon les lieux et les régions. Pour dépasser cette hétérogénéité, l'analyse des géographies rétentionnaires met en évidence des profils de réseau et des fonctionnements communs. Cependant, à chaque transfert les autorités mobilisent différents modes de transport. Interrogées sur les modalités concrètes de ces transferts, les autorités évoquent des formes spécifiques de mise à l'écart dessinant encore une multiplication des formes et des espaces d'enfermement.

\section{Les transferts entre les lieux d'enfermement et la multiplication de la mise à l'écart}

Les transferts nécessitent des modes de transports qui sont exclusivement réservés aux migrants (fourgons de police, bus privés loués pour l'occasion, etc.) ou inclus dans les 
transports en commun (ferries, bus, etc.). Dans cette dernière configuration, ils nécessitent une mise à l'écart supplémentaire au cours du voyage dont les modalités changent selon les lieux.

La police de l'île de Kos utilise les ferries qui font la liaison entre le continent et l'île pour transférer les migrants.

«Dans le bateau, on a un endroit pour les garder. C'est toujours le même, ce n'est pas un endroit spécifiquement fait pour ça, mais on le garde pour cette fonction. Les hommes ont les menottes, les femmes et les familles non. Autour d'eux, on met des barrières comme celles qu'il y a à l'aéroport " (le responsable du contrôle migratoire à la police de Kos, le 16 novembre 2012).

31 Le transfert de l'île de Kos au Pirée est l'occasion de la création de nouveaux espaces de mise à l'écart. La production d'enfermement en chaîne, au-delà de la question du respect des droits des migrants, implique l'enjeu de leur visibilité dans les espaces publics. Dans ce cas, les menottes et autres signes visibles de criminalisation des migrants contribuent à diffuser une image négative.

Un entretien auprès des mêmes autorités à Mytilène en 2012 met en lumière un autre fonctionnement. «Pour les envoyer à Athènes dans le ferry de la Blue Star, il y a un lieu pour les prisonniers. Sinon, dans les vieux bateaux, c'est dans une cabine» (le chef du service sécurité de la police de Mytilène, responsable du contrôle migratoire à Lesbos, juillet 2012).

33 La diversité des fonctionnements et du recours à ces micro-espaces d'enfermement prouve toute l'hétérogénéité des configurations d'enfermement en l'absence de législation claire les régissant. Dans la plupart des cas, la mise à l'écart est, au regard du contrôle migratoire, un enchaînement de mise à distance artificiellement créée par les autorités dans le but de séparer les migrants des habitants des espaces frontaliers.

Si les mobilités forcées dessinent des réseaux de lieux, elles mettent en lumière des réseaux d'acteurs qui peuvent influer sur les décisions de transfert et parfois limiter l'isolement des migrants dans les lieux d'enfermement.

\section{Le rôle des acteurs dans les réseaux de lieux d'enfermement}

Pour les avocats, travailleurs sociaux et militants, ces transferts sont une difficulté à prendre en compte dans leurs actions auprès des migrants. L'enjeu est donc d'avoir connaissance de ces transferts, de les anticiper ou de les rediriger. À propos du transfert des mineurs migrants non accompagnés, un avocat de la région d'Évros évoque des zones d'ombres :

"Le problème, c'est qu'on les perd. On ne peut pas les localiser après (le transfert). [La police ne vous informe pas de l'endroit où ils sont transférés ?] Si je demande, ils vont me dire, mais pas tout le temps parce que la police ici ne sait pas qui est l'autorité responsable. Donc, des fois, pour optimiser les transferts, peut-être qu'un bus de la police va venir de Feres et prendre toutes les personnes qui doivent être renvoyées à Alexandroupoli aux centres de détention. Mais cela ne signifie pas qu'une personne va aller à Feres, le bus peut les emmener au moins à Tichero, Soufli, Feres. Donc la police d'ici va dire "Feres est venu les chercher", mais on ne sait pas où ils vont. Et là, tu as 10 coups de fil à passer pour trouver où est la personne. C'est fou!» (le chef du service sécurité de la police de Mytilène, responsable du contrôle migratoire à Lesbos, juillet 2012).

Cet extrait d'entretien montre que les policiers chargés de la surveillance des lieux d'enfermement ont aussi parfois un faible degré de connaissance de ces transferts et 
n'en sont pas toujours les commanditaires. Les décisions sont aussi prises par la hiérarchie en fonction de la capacité des lieux et de leur spécialisation. Dans tous les cas, ces transferts nuisent à l'accompagnement juridique ou médical des migrants et peuvent être interprétés comme un outil de déstabilisation à l'image de ce que Nicholas Gill décrit dans le cas de la Grande-Bretagne où les transferts des demandeurs d'asile enfermés influent sur les déplacés eux-mêmes, sur les employés de ces lieux, et sur les supports militants possibles (Gill, 2009).

$37 \mathrm{Au}$ regard d'un tel fonctionnement, les liens entre intervenants dans les lieux militants et employés associatifs représentent une ressource importante. Les ONG intervenant dans les lieux d'enfermement, comme le Greek council for refugees (GCR), fonctionne déjà en réseau. Par exemple, à Évros Sud, deux avocats se partagent les quatre lieux d'enfermement. Ces affectations, qui suivent l'organisation policière, pallient les difficultés liées aux transferts et permettent une circulation de l'information. En dehors des employés, les militants, qui observent plus ou moins directement ce qui se déroule dans les lieux d'enfermement, peuvent se connaître et entretenir des liens entre localités ${ }^{22}$. L'existence de ces réseaux d'interconnaissance permet des mobilisations et des discussions autour des formes de luttes et des revendications possibles face au contrôle, mais peut aussi avoir un effet encore plus direct et impacter les transferts.

En 2009, je rencontre A., la coordinatrice d'Aghiassos, lieu d'accueil pour mineurs migrants non accompagnés de l'île de Lesbos. L'année d'après, je vais à Samos et rencontre G. qui connaît A. Elles étaient amies quand A. habitait Samos. Elles ont commencé à s'intéresser ensemble au sort des migrants sur l'île à l'époque où ils étaient enfermés dans un lieu improvisé au centre-ville de Vathy. Par la suite, A. a déménagé à Lesbos où elle devient responsable d'une structure d'accueil pour mineurs isolés. Régulièrement, A. va chercher au port de Mytilène des mineurs qui viennent de Samos, libéré après que G. ait indiqué aux autorités la possibilité d'un accueil à Aghiassos.

Les réseaux d'interconnaissance ne se limitent pas aux personnes exerçant la même activité. À la frontière, l'enquête l'a montré à plusieurs reprises : un militant des droits des migrants interrogé permet d'avoir un entretien avec un garde-côte de l'île, son cousin; une infirmière donne le contact d'une femme de ménage, etc. Ces réseaux occupent une place importante dans le lien entre l'intérieur des lieux d'enfermement et l'extérieur. Dans le cas où c'est un pouvoir discrétionnaire qui s'exerce dans les lieux à l'abri des regards, les liens d'interconnaissance permettent aux intervenants d'assister les migrants, de reconnecter les lieux à leur environnement, et de faire circuler l'information de ce qui s'y déroule. Le recrutement des travailleurs sociaux et avocats au sein de la population locale par les ONG mandatées pour intervenir dans les lieux d'enfermement constitue à ce titre un enjeu spécifique. Une employée d'ONG à Évros évoque ses bonnes relations avec la police: «Avec la police, ça se passe bien parce que je suis de Riza et que j'étais à l'école avec certains. Si j'avais été de Lesbos, cela aurait été plus difficile... » (un travailleur social, Évros, le 13 juin 2012).

Ces liens limitent la rupture induite par l'enfermement. Le fonctionnement des nouveaux lieux d'enfermement institutionnalisés avec l'emploi de personnel non local couplé à un fort turn-over des intervenants fait craindre une rupture possible de ces liens (Pillant, 2015). 


\section{Conclusion}

Dans les lieux d'enfermement, l'arbitraire policier s'impose aux migrants déjà soumis à de multiples incertitudes quant à leur avenir. L'utilisation policière de la confusion de l'espace et du temps de l'enfermement comme modalité répressive permet d'employer le terme de "gouvernance par l'incertitude " à l'échelle du lieu, terme développé par Kristen Sarah Biehl à l'égard du traitement turc des réfugiés syriens dans son pays (Biehl, 2015). L'enfermement constitue aussi un moment spécifique qui n'est pas toujours synonyme d'immobilité et les transferts fréquents renseignent le fonctionnement $\mathrm{du}$ contrôle migratoire au sein duquel les relations d'interconnaissance peuvent être décisives. Si l'enfermement prend sa source dans des mesures de mise à l'écart dès l'interpellation après franchissement irrégulier de la frontière, il est frappant de voir qu'il est systématiquement prolongé par une mobilité restreinte des migrants dans le pays après leur libération.

Après le contrôle, l'identification et l'enfermement à la frontière, deux situations administratives sont possibles: l'attribution d'une notification à quitter le territoire grec et la demande d'asile. Si la première situation permet de quitter la zone frontalière rapidement, elle est par la suite un puissant instrument de contrôle qui restreint la mobilité des migrants en Grèce. Bien que cette notification soit un moyen de régularisation temporaire (quelques jours à un mois), elle limite le séjour des migrants à certaines régions ${ }^{23}$. La deuxième situation, la demande d'asile, commence bien souvent dès la frontière et peut entraîner une période d'enfermement plus longue. Par la suite, elle confine les demandeurs en Grèce et limite leur accès à d'autres pays européens en raison de la réglementation Dublin.

Ces situations administratives représentent des possibilités d'extension du contrôle migratoire en dehors des lieux d'enfermement à l'échelle du pays. Ainsi, en déroulant le fil de la frontière, plus que de l'immobilité, ce sont des mobilités restreintes ou orientées qui se dessinent jusqu'au cœur du pays. L'enfermement des migrants est reproduit en chaîne représentant une mise en abyme du contrôle migratoire dont l'issue est plus qu'incertaine.

\section{BIBLIOGRAPHIE}

Baby-Collin V., 2014. Prendre place ici et là-bas ! Géographie multisituée des migrations boliviennes

(Argentine, États-Unis, Espagne). Habilitation à diriger des recherches à l'université Paris Nanterre La Défense.

Bathaïe.A, 2009. La Grèce, une étape cruciale dans le parcours migratoire des Afghans depuis la frontière iranienne jusqu'en Europe. Méditerranée, $\mathrm{n}^{\circ}$ 113, p 71-77.

Biehl K., 2015. Governing through uncertainty: experiences of being a refugee in Turkey as a transit country for asylum. Social Analysis, vol. 59, $\mathrm{n}^{\circ}$ 1, p. 57-75. 
Calavita K., 2005. Immigrants at the Margins: Law, Race, and Exclusion in Southern Europe. Cambridge University Press, $279 \mathrm{p}$.

$\mathrm{CPT}, 2013$. Report to the Government of Greece on the visit to Greece carried out by the European Committee for the Prevention of Torture and Inhuman or Degrading Treatment or Punishment (CPT). Conseil de l'Europe, Strasbourg. 51 p. https://rm.coe.int

Darley M., 2008. Frontière, asile et détention des étrangers : le contrôle étatique de l'immigration et son contournement en Autriche et en République tchèque. Thèse de doctorat à l'Institut d'Études Politiques de Paris.

Darley M., Lancelevée C., Michalon B., 2013. Où sont les murs ? Penser l'enfermement en sciences sociales. Cultures \& Conflits, vol. 90, p. 7-20.

Gemi E., Maroukis T., 2011. Circular migration between Albania and Greece: A case study. Rapport du projet Meitikos pour le European university institute, 39p. http://www.eui.eu/Projects/ METOIKOS/Documents/CaseStudies/METOIKOSCaseStudyGreeceAlbania.pdf

Gill N., 2009. Governmental mobility: The power effects of the movement of detained asylum seekers around Britain's detention estate. Political Geography, vol. 28, n 3, p. 186-196.

İçduygu A., 2011. The Irregular Migration Corridor between the EU and Turkey: Is it Possible to Block it with a Readmission Agreement? Research report, case study EU-US Immigration Systems. http:// hdl.handle.net/1814/17844

King R., DeBono D., 2013. Irregular Migration and the 'Southern European Migration Model' of Migration. Journal of Mediterranean Studies, vol. 22, n 1, p. 1-31.

Le Courant S., 2010. Ce que fait la politique de contrôle de l'immigration. Champ pénal, vol. VII, https://champpenal.revues.org/7889

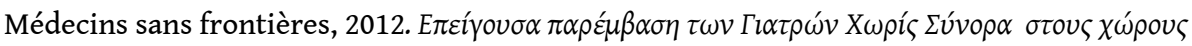
$\kappa \rho \alpha ́ \tau \eta \sigma \eta \varsigma \mu \varepsilon \tau \alpha v \alpha \sigma \tau \omega^{\prime} v \sigma \tau o v^{\prime} E \beta \rho o$. Rapport d'intervention à la frontière gréco-turque à Évros, $20 \mathrm{p}$. https://www.msf.gr/sites/default/files/msfpublications/

report_detention_migrants_greece_gr.pdf

Michalon B., 2012. La mobilité au service de l'enfermement ? Géographie et cultures, n 81 , p. 91-110.

Milhaud O., 2009. La clôture suffit-elle à faire un espace d'enfermement ? Spatialités contradictoires et poreuses des prisons françaises contemporaines. Les cahiers de l'ADES, $\mathrm{n}^{\circ} 4$, p. $45-58$.

Papadopoulou-Kourkoula A., 2008. Transit Migration. Palgrave Macmillan, 224 p.

Pérouse J.-F., 2002. Migrations, circulations et mobilités internationales à Istanbul, Dossier de l'IFEA, $\mathrm{n}^{\circ}$ 13, p. 9-29.

Pillant L., 2014. Introduction of procedures for migration controls in Greece. Case study in the Aegean Islands. In Anteby L., Baby-Collin V., Mazzella S., Mourlane S., Parizot C., Regnard C., Sintès P. (collectif MIMED) dir., Borders, Migrations and mobilities. Perspectives from the Mediterranean. Editions Peter Lang, $310 \mathrm{p}$.

Pillant L., 2015. Les conséquences socio-spatiales des nouvelles modalités du contrôle migratoire à la frontière gréco-turque. L'Espace Politique, n² 25. http://espacepolitique.revues.org.lama.univamu.fr/3369

Pillant L., 2017. La frontière comme assemblage. Géographie critique du contrôle migratoire à la frontière orientale de la Grèce. Thèse de doctorat en géographie à l'Université Aix-Marseille. 
de Rapper G., 2002. Espace et religion : chrétiens et musulmans en Albanie du Sud. Études balkaniques, $\mathrm{n}^{\circ}$ 9, $\mathrm{p}$ 17-39.

Sélimanovski C., 2009. Effets de lieu et processus de disqualification sociale. Espace populations sociétés. Espace Populations et Sociétés, $\mathrm{n}^{\circ}$ 1, p. 119-133.

Schmoll C., Bernardie-Tahir N., 2015. Iles, frontières et migrations méditerranéennes : Lampedusa et les autres. L'Espace Politique, $\mathrm{n}^{\circ} 25$. http://espacepolitique.revues.org/3333

Sintès P., 2001. La migration des Albanais en Grèce. Difficultés méthodologiques pour une étude géographique. REMI, $\mathrm{n}^{\circ}$ 17, p 67-85.

Tassin L., 2016. Les frontières de la rétention : genre et ethnicité dans le contrôle des étrangers en instance d'expulsion. Critique internationale, $\mathrm{n}^{\circ} 72$, $\mathrm{p}$ 35-52.

Topaz O.E., 2014. Surveillance, subjectivity and resistance at the frontiers of Europe : a matérialist analysis of the Greece-Turkey borders. Thèse de doctorat à la Queen's University, Kingston, Ontario, Canada.

\section{NOTES}

1. Selon Aspasia Papadopoulou-Kourkoula, la frontière orientale de la Grèce était déjà un lieu de passage pour les migrants dans les années 1990.

2. En 2003, la police interpelle 2431 franchissements irréguliers, ils seront 52484 en 2010.

3. Le règlement Dublin II en 2003 détermine le pays de l'Union européenne responsable de l'enregistrement de la demande d'asile. Jusqu'en 2011, date de la suspension des renvois Dublin en Grèce grâce au jugement de la Cour européenne des droits de l'homme (CEDH), la demande d'asile en Grèce nécessitait un maintien du requérant dans le pays de l'enregistrement.

4. Cette loi institue l'expulsion comme politique migratoire jusqu'alors focalisée sur une immigration depuis les pays limitrophes (Sintès, 2001 ; de Rapper, 2002).

5. L'enfermement est entendu ici comme la privation de liberté d'un étranger en raison de son irrégularité administrative dans le pays dans lequel il se trouve.

6. Bien que l'Albanie signe avec la communauté européenne un accord de réadmission en 2005 (Maroukis et Gemi, 2011, p. 11), la Grèce pratique l'expulsion vers ce pays depuis les années 1990.

7. La Grèce n'a un accord de réadmission qu'avec la Turquie en dehors de l'UE (Source site internet de Migreurop consulté le 28/03/2017 http://www.migreurop.org/article1591.html ).

8. Les accords de réadmission concernent les ressortissants de pays avec lesquels la Turquie a des accords de réadmission - Nigeria, Pakistan - (Içduygu, 2011) et ses pays limitrophes - Iran, Irak, Géorgie, Syrie - (Topaz, 2014, p. 184). Les expulsions potentielles vers la Turquie concernent ces nationalités (les Syriens jusqu'en 2011).

9. Le maximum légal est de trois mois jusqu'en 2011, où la période s'étend à 18 mois, comme dans le reste des pays de l'UE.

10. 18 lieux improvisés, 14 institutionnalisés et 24 intermédiaires (inventaire réalisé grâce aux archives du ministère de l'Intérieur, du travail de mémoire de Ioulia Iliadi et des différents terrains de thèse).

11. Transposition de la directive européenne de retour qui étend la durée légale d'enfermement et favorise l'éloignement du territoire européen.

12. 54 entretiens ont été réalisés avec la police, les gardes-frontière, l'armée et Frontex ; 79 avec des avocats, des infirmiers, des femmes de ménage; 47 avec des maires, des préfets, et des fonctionnaires.

13. 21 entretiens ont été réalisés avec des migrants. 
14. 175 documents analysés au ministère.

15. Une récolte de document a été effectuée dans le lieu de Venna à Évros et de Mersinidi à Chios île au Sud de Lesbos.

16. Vathy est la capitale de l'île de Samos, le lieu est localisé en périphérie de la ville.

17. Observation à Vathy durant la manifestation en dehors du lieu le 17 avril 2010.

18. Notamment révélé par le rapport du CPT, 2013, p. 54.

19. Les archives ont permis l'analyse de Lesbos, Chios et Samos en 2009, Évros en 2012 et le Dodécanèse de 2008 à 2012. Ces différentes dates sont comparables dans la mesure où ce sont des périodes d'arrivées nombreuses de migrants. Les archives sont constituées de factures de transport et de correspondances entre les préfectures, la police et le ministère concernant ces transferts.

20. Le Dodécanèse est aussi un cas de réseau multipolarisé (Pillant, 2017, p. 435)

21. Entretien avec un avocat de l'UNHCR, le 12/07/2012.

22. Les rassemblements militants sur la question spécifique du contrôle migratoire (No Border à Lesbos, rencontre à Athènes), ou des manifestations moins spécifiques (festival antiraciste de Samos, Athènes ou Chios) jouent un rôle important dans ces rencontres.

23. Les préfectures d'Archaïe et de Thesprotia dans lesquelles se trouvent les ports de Patras et Igoumenitsa sont interdites aux migrants ayant cette notification.

\section{RÉSUMÉS}

Le contrôle migratoire à la frontière orientale de la Grèce repose principalement sur l'enfermement des migrants ayant franchi la frontière sans autorisation. Dans les années 2000 émergent des lieux d'enfermement peu réglementés, aux fonctionnements hétérogènes. Cet article explore les modalités de contrôle des autorités sur les migrants dans ces lieux. Celui-ci consiste notamment en une incertitude spatio-temporelle qui renforce le caractère répressif et dissuasif de la privation de liberté. Ces incertitudes ne sont pas uniquement à l'échelle du lieu mais se doublent de transferts fréquents qui peuvent survenir à tout moment. En suivant les mobilités forcées des migrants, il s'agit d'appréhender la fonction des lieux d'enfermement, leur multiplication, ainsi que leur connexion en réseau.

Migration control at Greece's eastern border is mainly based on the detention of migrants who have crossed the border without authorisation. In the 2000s, unregulated detention places with heterogeneous functions emerged. This article explores how the authorities can control migrants in these places. It consists in particular of a spatio-temporal uncertainty that reinforces the repressive and dissuasive character of liberty deprivation. These uncertainties are not limited to the scale of the place, but are combined with frequent transfers that can occur at any time. By following the forced migrants' mobilities, this article examines the detention places functions, their multiplication and their network connections.

\section{INDEX}

Keywords : detention place, migration control, transfer, network, border, Greece

Mots-clés : lieu d'enfermement, contrôle migratoire, transfert, réseau, frontière, Grèce 


\section{AUTEUR}

\section{LAURENCE PILLANT}

Laurence Pillant, 1.pillant@gmail.com, est Docteure en géographie, chercheuse associée au laboratoire TELEMME, UMR 7303 - MMSH, AMU. Elle a publié récemment :

- Pillant L., 2016. La Grèce : une crise migratoire chronique. Plein droit, vol. 4, n 11, p. 31-34.

- Pillant L., Tassin, L., 2016. Lesbos, l'île aux grillages. Migrations et enfermement à la frontière grécoturque. Cultures \& Conflits, 99-100, mis en ligne le 16 février 2017. http://conflits.revues.org/ 19068

- Pillant L., 2015. Les conséquences socio-spatiales des nouvelles modalités du contrôle migratoire à la frontière gréco-turque. L'Espace Politique, vol. 25, n 1, mis en ligne le 05 avril 2015. http:// espacepolitique.revues.org/3369

- Pillant L., 2014. Introduction of procedures for migration controls in Greece. Case study in the Aegean Islands. In Anteby L., Baby-Collin V., Mazzella S., Mourlane S., Parizot C., Regnard C., Sintès P. (collectif MIMED) dir., Borders, Migrations and mobilities. Perspectives from the Mediterranean, Editions Peter Lang, 310 p. 